\title{
MODELO DE TRANSFORMADOR COM TAP VARIÁVEL ADEQUADO PARA ESTUDOS DE ESTABILIDADE DE TENSÃO
}

\author{
Carlos Aparecido Ferreira* \\ carlosaparecidodeletrobras.com
}

\author{
Ricardo Prada* \\ pradadele.puc-rio.br
}

\author{
*PUC-Rio / Departamento de Engenharia Elétrica \\ Rua Marquês de São Vicente, 225, Edifício Cardeal Leme, sala 401 \\ Gávea, Rio de Janeiro - RJ, 22453-900
}

\begin{abstract}
Tap-Changing Transformer Model for Voltage Stability Analysis

The model for tap-changing transformers currently in widespread use consists of an impedance, measured in a short-circuit test with a nominal tap, in series with an ideal transformer. The use of this model in voltage stability studies leads to qualitatively incorrect results. For demonstration purpose a small circuit and the concepts of maximum load, equivalent load impedance and voltage control effects are used. An improved model that takes into account laboratory results, circuit laws and voltage stability theory is proposed. Using IEEE test systems, different results are shown for the conventional and proposed models.
\end{abstract}

KEYWORDS: LTC, OLTC, ULTC, Tap-Changing Transformer, Steady-State Model, Voltage Stability.

\section{RESUMO}

O modelo de transformador com tap variável utilizado mundialmente consiste de uma impedância, obtida através do ensaio em curto-circuito e com tap nominal, em série com um transformador ideal. Em estudos de estabilidade de tensão, o uso desse modelo leva a resultados qualitativamente errados. Para demonstração, utiliza-se um circuito pequeno e os conceitos de máxima potência transmitida, impedância equi-

Artigo submetido em 25/07/2011 (Id.: 1362)

Revisado em 28/09/2011, 09/11/2011, 09/11/2011

Aceito sob recomendação do Editor Associado Prof. Julio Cesar Stacchini Souza valente da carga, e efeito do controle de tensão. Propõe-se um novo modelo coerente com os resultados obtidos em laboratório, com as leis de circuitos elétricos e com a teoria de estabilidade de tensão. Com sistemas-teste do IEEE, são mostrados resultados diferentes comparando-se os modelos usual e proposto.

PALAVRAS-CHAVE: LTC, OLTC, ULTC, Transformador com Tap Variável, Modelo em Regime Permanente, Estabilidade de Tensão.

\section{INTRODUÇÃO}

A representação de equipamentos elétricos através de simples combinações de resistências e reatâncias há muito tempo vem sendo utilizada (MacLeod, 1929). Obviamente, porém, um circuito elétrico equivalente tem suas limitações na representação de um dispositivo ou equipamento Assim, o circuito elétrico representa uma aproximação do comportamento elétrico, magnético e até mecânico (no caso de máquina girante) do equipamento.

Determinado modelo de equipamento elétrico não é necessariamente o único ou o mais conveniente modo de se representar o equipamento para todas as situações, devendo ser escolhido o modelo que melhor atende ao problema analisado (Boyajian, 1930). Entretanto, a maior ou menor familiaridade com um ou mais modelos, é um fator determinante nessa escolha. Essa é uma explicação para a demora em se utilizar excelentes ferramentas, como foi o caso do método das componentes simétricas no início do século passado (Boyajian, 1930). 
Modelar transformador com tap variável adequadamente é fundamental em análises de estabilidade de tensão, tanto no que diz respeito às informações fornecidas ao operador referentes às margens de estabilidade de tensão, quanto aos efeitos de ações de controle de tensão.

O objetivo deste artigo é apresentar falhas do modelo usual de transformador com tap variável e propor um novo modelo. Poderá ser aplicado em qualquer estudo em regime permanente.

\section{CONCEITOS BÁSICOS}

\subsection{Estabilidade de Tensão}

Na Figura 1 apresenta-se uma típica curva $\varphi$ (ângulo do fator de potência) constante no plano $S V$, também conhecida como "curva-nariz" ou "curva do nariz". A curva foi traçada para uma barra $k$ qualquer, onde $\mathrm{S}_{i}$ refere-se à potência aparente injetada na barra, para determinado ponto de operação. Nessa situação, tem-se uma solução na região normal (ponto $\mathrm{A}$, com tensão $\mathrm{V}_{k}$ elevada e baixa corrente) e outra na região anormal (ponto $\mathrm{B}$, com tensão $\mathrm{V}_{k}$ baixa e elevada corrente). $\mathrm{O}$ ponto $\mathrm{C}$ corresponde à máxima potência aparente injetada na barra $\left(\mathrm{S}_{m}\right)$.

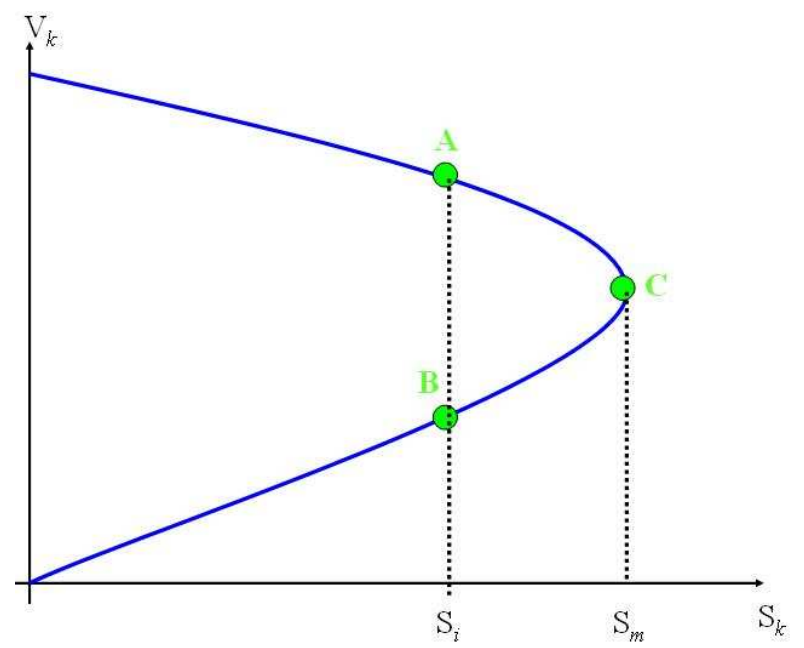

Figura 1: Curva $\varphi$ Constante no Plano SV

\subsubsection{Impedância Equivalente no Ponto de Má- ximo Carregamento}

Supondo que o gráfico apresentado na Figura 1 é referente ao sistema de 2 barras apresentado na Figura 2, onde a carga é modelada como impedância constante, a curva pode ser obtida variando-se a carga de $\mathrm{Z}_{1}=\infty$, onde $\mathrm{V}_{1}=\mathrm{V}_{0}$, até $\mathrm{Z}_{1}=$ 0 , onde $\mathrm{V}_{1}=0$. De acordo com (Close, 1975), o módulo da impedância da carga no ponto de máximo carregamento vale $Z_{t}$. Ou seja, quando o módulo da impedância da carga $Z_{1}$ for igual ao módulo da impedância da linha de transmissão $\mathrm{Z}_{t}$, a potência transmitida para a carga é a máxima $\left(S_{m}\right)$.

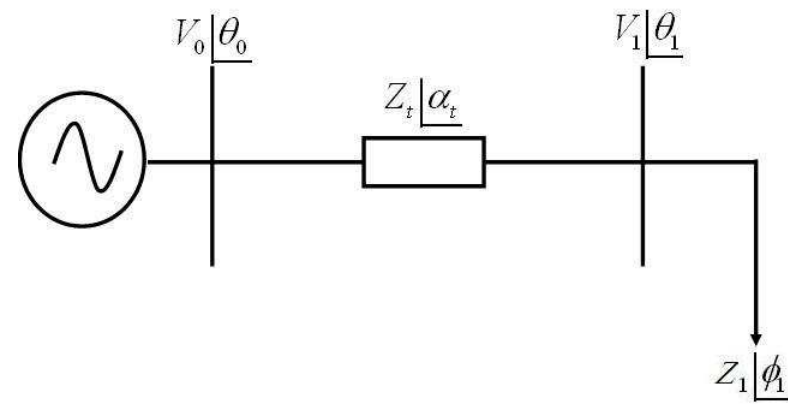

Figura 2: Sistema de 2 Barras

Por outro lado, se a representação do circuito de 2 barras for genérica, com elementos em paralelo com a fonte e com a carga, conforme mostrado na Figura 3, obtendo-se o circuito equivalente de Thevenin visto da carga, apresentado na Figura 4, o mesmo raciocínio pode ser utilizado. Nesse caso, então, analogamente ao que foi apresentado no sistema de 2 barras da Figura 2, o módulo da impedância da carga no ponto de máximo carregamento será $\mathrm{Z}_{t h}$. Ou seja, quando o módulo da impedância da carga $Z_{1}$ for igual ao módulo da impedância equivalente de Thevenin vista dos terminais da carga $Z_{t h}$, a potência transmitida é máxima $\left(S_{m}\right)$.

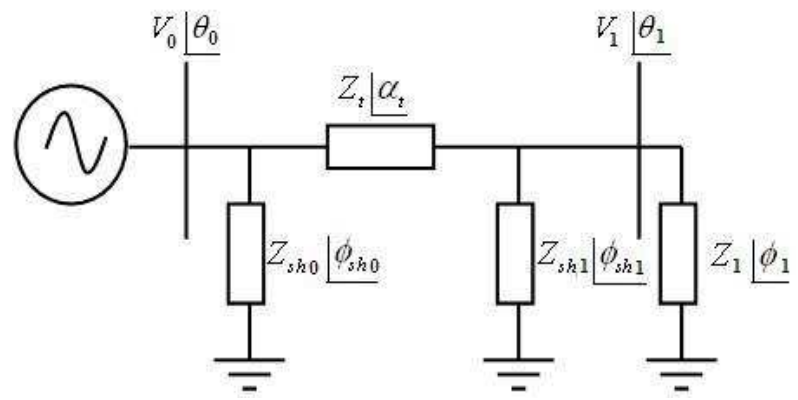

Figura 3: Sistema Genérico de 2 Barras

\subsubsection{Ações para Aumento da Capacidade de Transmissão e de Controle de Tensão}

Considerando-se que foi inserido um capacitor na barra de carga do circuito apresentado na Figura 2, tem-se uma nova curva $\varphi$ constante no plano SV. Na Figura 5, observa-se que a introdução do capacitor faz com que o máximo carregamento aumente. Pode ser observado ainda que, se o sistema opera 


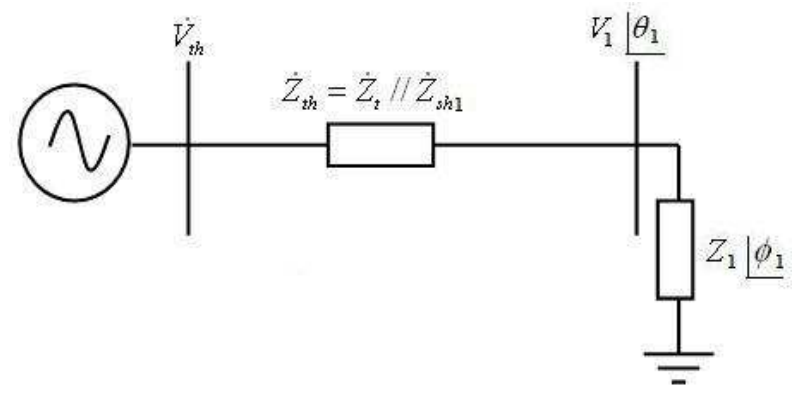

Figura 4: Equivalente de Thevenin do Sistema Genérico de 2 Barras

no ponto de operação A (região normal) e é inserido um capacitor no circuito com o objetivo de se elevar a tensão, essa ação de controle tem efeito esperado tanto para carga potência constante (novo ponto de operação será A') quanto para carga impedância constante (novo ponto de operação será A").

Por outro lado, conforme apresentado na Figura 6, se o sistema opera no ponto de operação B (região anormal) e é inserido um capacitor no circuito com o objetivo de se elevar a tensão, essa ação de controle tem efeito oposto ao esperado para carga potência constante (novo ponto de operação será B'), ou seja, a tensão diminui quando o capacitor é inserido. Se o modelo da carga é impedância constante, porém, a ação de controle terá efeito esperado (novo ponto de operação ponto de operação será B").

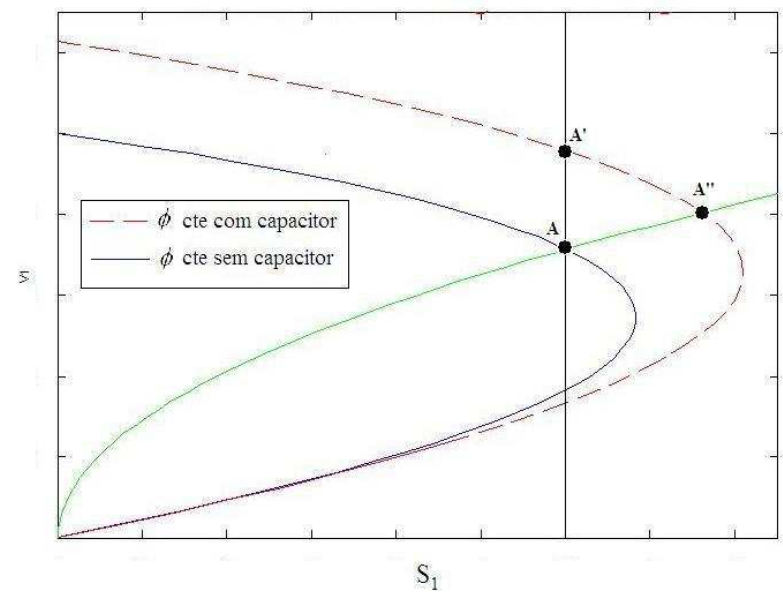

Figura 5: Análise da Ação de Controle de Tensão com Ponto de Operação na Região Normal

A inserção de capacitor foi apenas um exemplo de ação de controle de tensão e de aumento da máxima capacidade de transmissão. Outras ações devem ter os mesmos resultados

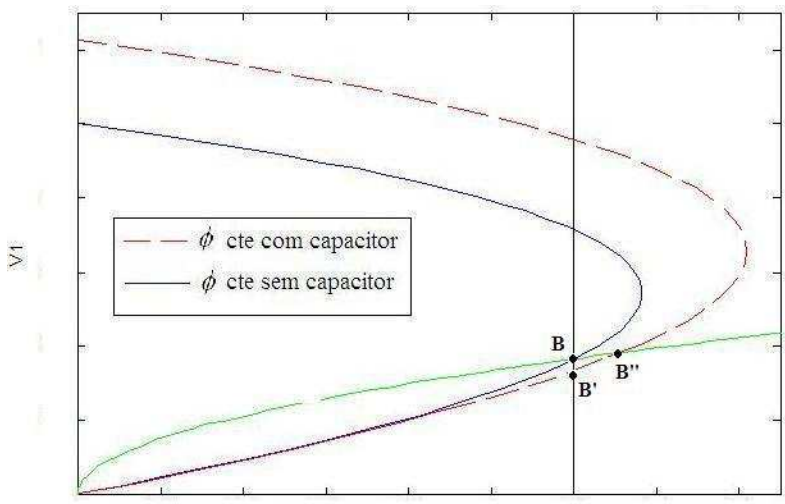

$\mathrm{S}_{1}$

Figura 6: Análise da ação de Controle de Tensão com Ponto de Operação na Região Anormal

qualitativos apresentados, tais como aumento do valor de $\mathrm{V}_{0}$, inserção de outra linha de transmissão em paralelo à existente, inclusão de compensação série capacitiva e inclusão de transformador com troca de taps, como será abordado neste artigo.

\subsection{Modelo de Transformador em Regime Permanente}

Na Figura 7 apresenta-se o modelo de transformador operando em regime permanente, onde é desprezada a impedância composta pela reatância de magnetização e pela resistência referente às perdas no ferro, devido ao seu elevado valor comparado à impedância dos enrolamentos $\dot{Z}$ (Staff of MIT, 1965). Esta impedância, referida a determinado lado do transformador, pode ser determinada através do teste de curto-circuito (Staff of MIT, 1965) e, em ohms, é dada por:

$$
\dot{Z}(\Omega)=\dot{Z}_{k}^{\prime}(\Omega)+\dot{Z}_{m}(\Omega)
$$

onde:

$$
\dot{Z}_{k}^{\prime}(\Omega)=\dot{Z}_{k}(\Omega)\left(\frac{N_{m}}{N_{k}}\right)^{2}
$$

Na Figura 7, o valor da impedância está referido ao lado $m$, sem perda de generalidade. Em p.u., os valores das impedâncias referidas aos lados $k$ ou $m$ terão os mesmos valores, desde que, na escolha dos valores-base de tensão de cada lado do transformador, seja obedecida a relação de transformação. E ainda, a potência-base deve ser a mesma nos dois lados do transformador, assim como em todo o sistema (Grainger e Stevenson, 1975). 


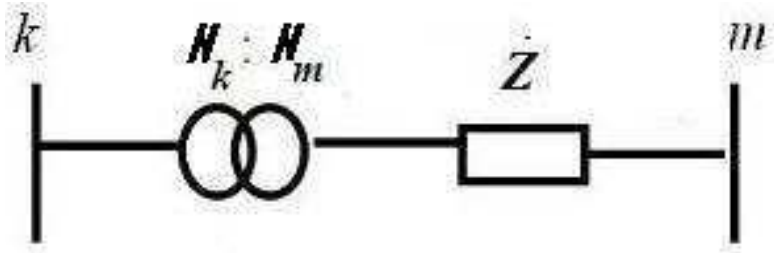

Figura 7: Modelo de Transformador em Regime Permanente

Assim, o transformador pode ser representado completamente por sua impedância em p.u., sem o transformador ideal, conforme mostrado na Figura 8, já que nenhuma transformação é necessária. A corrente terá o mesmo valor em p.u. nos dois lados. Essa é uma vantagem da representação em p.u.

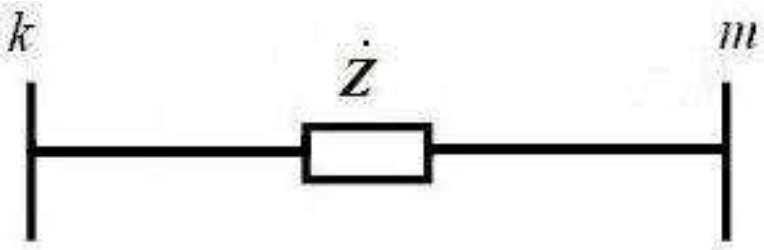

Figura 8: Representação de Transformador em p.u. Quando Valores-Base de Tensão Obedecem a Relação de Transformação

O problema é que, na operação de sistemas elétricos de potência, é comum variar-se taps de transformadores com o objetivo de controlar o perfil de tensão. Nessa situação, seria necessário estabelecer novas bases de tensão e de impedância no lado do sistema onde o tap foi mudado e recalcular todas as grandezas em p.u., de forma a ser observada a vantagem da representação em p.u. mencionada. Assim, tensões nodais e impedâncias de linhas de transmissão e de transformadores deveriam ser modificadas a cada alteração do tap. Esse procedimento, evidentemente, é inviável.

\section{MODELO USUAL DE TRANSFORMA- DOR COM TAP VARIÁVEL}

\subsection{Representação do Modelo Usual}

Para solucionar o problema descrito, o transformador ideal é reintroduzido na representação de transformador com tap variável, conforme apresentado na Figura 9. Este modelo é mundialmente utilizado.

Além da impedância, o tap também é representado em p.u. na Figura 9, sendo o tap-base igual ao tap nominal, que é igual à relação entre o número de espiras $\left(\mathrm{N}_{m}: \mathrm{N}_{k}\right)$, em con-

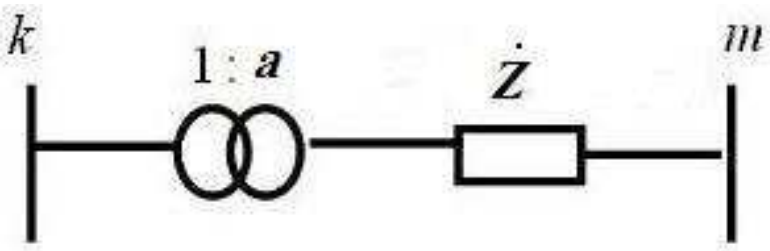

Figura 9: Modelo Usual Representado através de Transformador Ideal em Série com Impedância

dições nominais. Assim, $a=1$ quando a relação é a nominal, com o circuito da Figura 9 igual ao da Figura 8. As tensõesbase permanecem constantes, obedecendo a relação nominal de transformação, independente do valor do tap.

Como mencionado, a impedância $\dot{Z}$ é medida através do teste de curto-circuito tendo, portanto, um valor que é dependente da posição do tap para a qual o teste foi realizado (Monticelli e Garcia, 2003). Entretanto, o valor da impedância utilizado é correspondente ao tap nominal, o que é justificado pela pequena variação do tap em torno de 1 p.u.

Para eliminar o transformador ideal do modelo apresentado na Figura 9, de forma a facilitar a montagem da matriz de admitância nodal, o circuito elétrico é representado por seu $\pi$ equivalente, conforme mostrado na Figura 10 e em (MacLeod, 1929; Boyajian, 1929; Boyajian, 1930; Clarke, 1950). Esse circuito foi inicialmente apresentado em (MacLeod, 1929 e Boyajian, 1929).

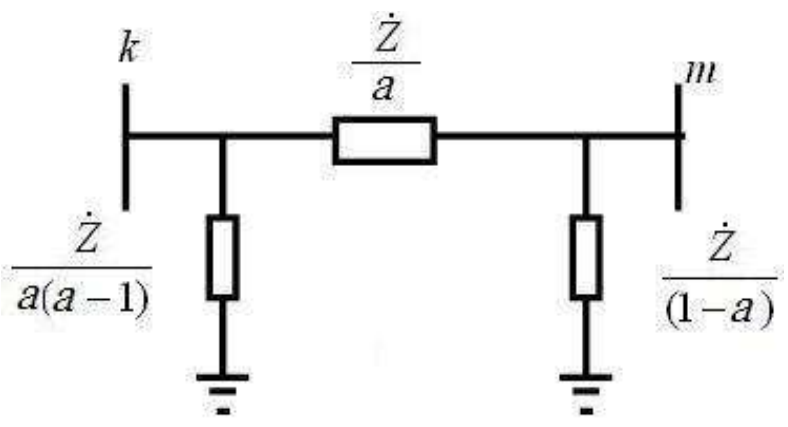

Figura 10: Modelo Usual Representado através do Circuito П Equivalente

\subsection{Modelo Usual de Transformador com Tap Variável em Estudos de Estabili- dade de Tensão}

Considerando-se a representação usual de transformador com tap variável, conforme mostrado nas Figuras 9 e 10, observa-se que se gerador está conectado à barra $k$ e carga na 
barra $m$, a máxima transferência de potência ocorre quando $Z_{m}=Z_{t h}=Z$, conforme apresentado na Seção 2.1.1, onde $Z_{m}$ é a impedância equivalente da carga, $Z_{t h}$ é a impedância de Thevenin vista da barra $m$ e Z é a impedância total do transformador (no lado $m$ ), todas em p.u. Nesse caso, observa-se que a impedância no ponto de máximo carregamento não é modificada quando o tap é alterado.

Por outro lado, invertendo-se o sentido do fluxo de potência, ou seja, com o gerador na barra $m$ e carga na barra $k$, a máxima transferência de potência ocorre quando $Z_{k}=Z_{t h}=$ $\frac{Z}{a^{2}}$, onde $Z_{k}$ é a impedância equivalente da carga e $\frac{Z}{a^{2}}$ é a impedância total do transformador refletida para o lado $k$. Nessa situação, ao contrário do caso anterior, observa-se que a impedância no ponto de máximo carregamento é modificada quando o tap é alterado.

Considerando-se, ainda, os circuitos usuais apresentados nas Figuras 9 e 10, pode-se traçar as curvas para $\varphi$ constante no plano SV para dois valores diferentes de taps do transformador, assim como a curva da impedância equivalente da carga que passa pelos pontos de máximo carregamento. Verificase na Figura 11 que, se o fluxo de potência flui da barra $k$ para a barra $m$, ao alterar o tap do transformador, a impedância equivalente da carga no ponto de máximo carregamento permanece a mesma, como já dito. Entretanto, o ponto de máximo carregamento varia.

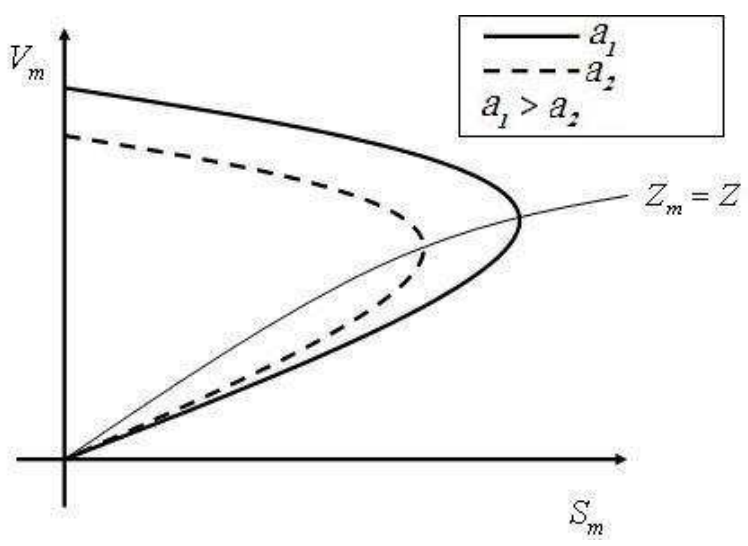

Figura 11: Curvas $\varphi$ e Z Constante para Dois Valores de Tap com o Modelo Usual e com Fluxo da Barra $k$ para a barra $m$

Por outro lado, conforme mostrado na Figura 12, se o fluxo de potência vai da barra $m$ para a barra $k$, ocorre o oposto, ou seja, o ponto de máximo carregamento permanece o mesmo e a impedância equivalente da carga no ponto de máximo carregamento varia.

Curvas, como a da Figura 12 são apresentadas em (Baracho e Café, 1991; El-Sadek et alii, 1999). Em (IEEE/PES, 2002) também é indicado que o ponto de máximo carregamento permanece constante com a variação do tap. Esse resultado é qualitativamente inconsistente com o que foi apresentado na Seção 2.1.2.

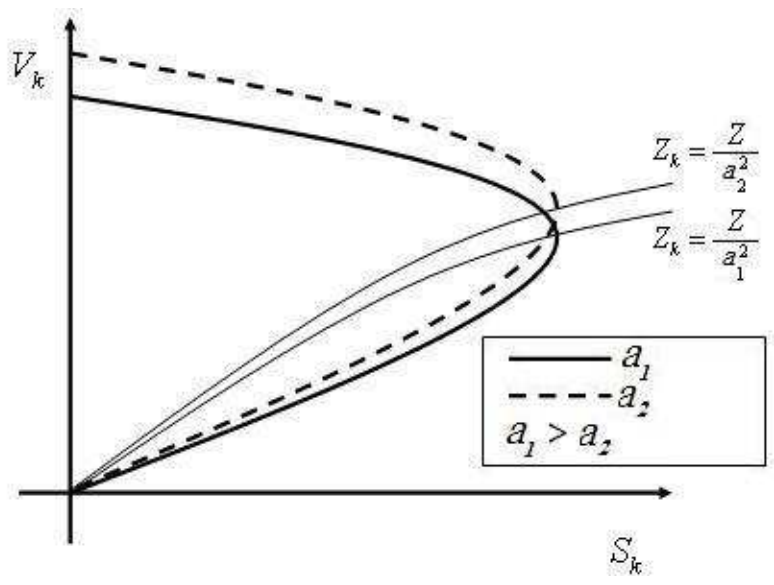

Figura 12: Curvas $\varphi$ e Z Constantes para Dois Valores de Tap com o Modelo Usual e com Fluxo da Barra $m$ para a barra $k$

É inesperado que o sentido do fluxo de potência tenha como consequência as diferenças citadas. Conjeturou-se que, tanto o ponto de máximo carregamento, quanto a impedância equivalente da carga neste ponto, variariam com o tap, independente do sentido do fluxo de potência. Essa intuição foi comprovada através de ensaios em laboratório com um transformador real com tap variável (Ferreira e Prada, 2009), apresentados no Apêndice A. Assim, o modelo de transformador com tap variável utilizado mundialmente em programas de fluxo de potência apresenta resultados qualitativamente incorretos em estudos de estabilidade de tensão.

\section{MODELO PROPOSTO DE TRANSFOR- MADOR COM TAP VARIÁVEL}

\subsection{Representação do Modelo Proposto}

$\dot{Z}_{k}(\Omega)$ e $\dot{Z}_{m}(\Omega)$, apresentados em (1) e (2), são as impedâncias em ohms referentes aos enrolamentos de cada um dos lados do transformador. Essas impedâncias não podem ser obtidas separadamente, mesmo que fossem realizados dois testes de curto-circuito, um em cada lado do transformador, devido à dependência linear entre as duas equações geradas. Porém, de acordo com (Westinghouse, 1964; Staff of MIT, 1965; Del Toro, 1990):

$$
\dot{Z}_{k}^{\prime}(\Omega)=\dot{Z}_{m}(\Omega)=\frac{\dot{Z}(\Omega)}{2}
$$

Assim: 


$$
\dot{Z}_{k}(\Omega)=\frac{\dot{Z}(\Omega)}{2}\left(\frac{N_{k}}{N_{m}}\right)^{2}
$$

onde, $\dot{Z}(\Omega)$, como mencionado, é obtida através do teste de curto-circuito com tap nominal.

Por outro lado, as impedâncias-base são dadas por:

$$
\begin{gathered}
Z_{\text {base }_{m}}=\frac{\left(V_{\text {base }_{m}}\right)^{2}}{S_{\text {base }}} \\
Z_{\text {base }_{k}}=\frac{\left(V_{\text {base }_{m}}\right)^{2}}{S_{\text {base }}}\left(\frac{N_{k}}{N_{m}}\right)^{2}
\end{gathered}
$$

Verifica-se facilmente que as impedâncias $\dot{Z}_{k}(\Omega)$ e $\dot{Z}_{m}(\Omega)$ guardam entre si relação quadrática de transformação, assim como as impedâncias-base dos lados $k$ e $m$. Logo, as impedâncias de cada lado do transformador em p.u. são iguais:

$$
\dot{Z}_{k}(\text { p.u. })=\dot{Z}_{m}(\text { p.u. })=\frac{\dot{Z}(\Omega)}{2} \frac{S_{\text {base }}}{\left(V_{\text {base }_{m}}\right)^{2}}
$$

ou

$$
\dot{Z}_{k}(p . u .)=\dot{Z}_{m}(p . u .)=\frac{\dot{Z}}{2}
$$

Tem-se, então, o modelo proposto apresentado na Figura 13, onde não há reflexão de impedância para um dos lados.

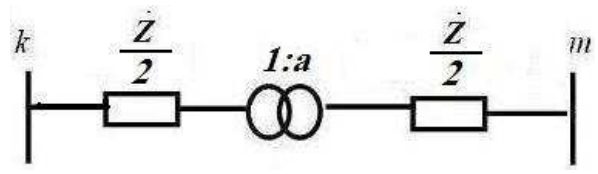

Figura 13: Modelo Proposto

O modelo proposto, como apresentado, considera as impedâncias em cada enrolamento do transformador. Se a impedância do lado $k$ do circuito na Figura 13, for refletida para o lado $m$, considerando-se a relação $1: a$, a impedância total variará quando o tap é modificado, ao contrário do que ocorre com o modelo usual, acarretando em diferenças qualitativas entre os resultados obtidos pelos dois modelos, como será mostrado.

Observa-se ainda que a impedância total no lado $m$ será igual a $\dot{Z}$ somente quando a relação de transformação for a nominal $(\mathrm{a}=1)$ e, nesse caso, o modelo proposto coincide com o modelo usual.

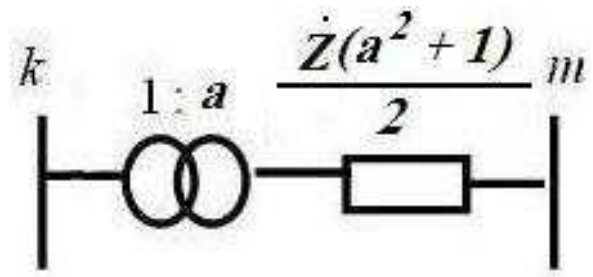

Figura 14: Modelo Proposto com Reflexão de Impedância

Na Figura15 apresenta-se o circuito $\pi$ equivalente ao modelo proposto.

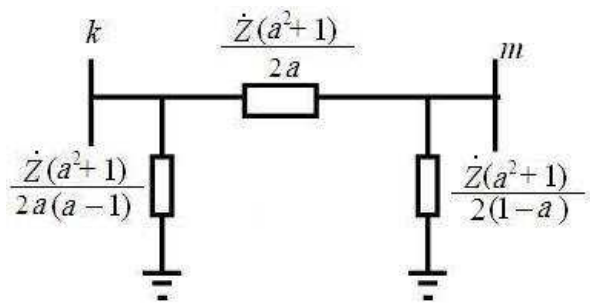

Figura 15: Modelo Proposto Representado através do Circuito П Equivalente

\subsection{Modelo Proposto de Transformador com Tap Variável em Estudos de Es- tabilidade de Tensão}

Considerando-se o modelo proposto de transformador com tap variável, conforme mostrado nas Figuras 13, 14 e 15, observa-se que, se gerador está conectado à barra $k$ e carga na barra $m$, a máxima transferência de potência ocorre quando $Z_{m}=Z_{t h}=Z\left(a^{2}+1\right) / 2$, onde $Z_{m}$ é a impedância equivalente da carga e $Z\left(a^{2}+1\right) / 2$ é a impedância do transformador do lado $m$ mais a impedância do lado $k$ refletida para o lado $m$. Nesse caso, observa-se que a impedância no ponto de máximo carregamento é modificada quando o tap é alterado, ao contrário do que ocorre com o modelo usual.

Invertendo-se o sentido do fluxo de potência, ou seja, com o gerador na barra $m$ e carga na barra $k$, a máxima transferência de potência ocorre quando $Z_{k}=Z_{t h}=Z\left(a^{2}+1\right) /\left(2 a^{2}\right)$, onde $Z_{k}$ é a impedância equivalente da carga e $\mathrm{Z}\left(\mathrm{a}^{2}+1\right) /\left(2 \mathrm{a}^{2}\right)$ é a impedância do transformador do lado $k$ mais a impedância do lado $m$ refletida para o lado $k$. Nessa situação, observa-se que a impedância no ponto de máximo carregamento também é modificada quando o tap é alterado.

Considerando-se, ainda, os circuitos das Figuras. 13, 14 e 15 , pode-se traçar as curvas para $\varphi$ constante no plano SV para dois valores diferentes de taps do transformador com 
tap variável, assim como a curva da impedância equivalente da carga que passa pelos pontos de máximo carregamento. Verifica-se na Figura 16 que, se o fluxo de potência flui da barra $k$ para a barra $m$, ao alterar o tap do transformador, tanto a impedância equivalente da carga no ponto de máximo carregamento, quanto o próprio ponto de máximo carregamento, variam quanto o tap é alterado, como esperado. Utilizando-se o modelo usual, a impedância equivalente da carga permanece constante quando o tap é alterado, como observou-se na Figura 11.

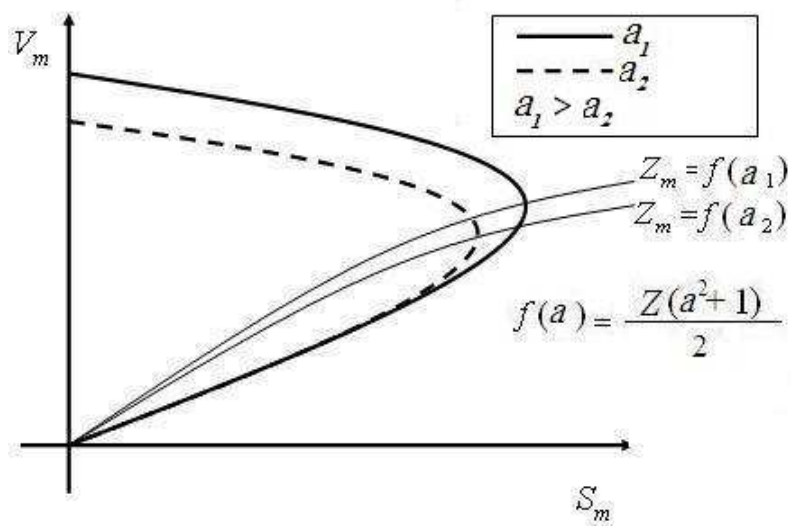

Figura 16: Curvas $\varphi$ e Z Constante para Dois Valores de Tap com o Modelo Proposto e com Fluxo da Barra $k$ para a Barra $m$

Conforme mostrado na Figura17, se o fluxo de potência flui da barra $m$ para a barra $k$, novamente, tanto o ponto de máximo carregamento, quanto a impedância equivalente da carga neste ponto variam quando o tap é alterado, como esperado. Utilizando-se o modelo usual, o ponto de máximo carregamento permanece constante quando o tap é alterado, como observou-se na Figura 12. Ressalta-se que os resultados obtidos utilizando o modelo proposto são qualitativamente coerentes com o que foi apresentado na Seção 2.1.2.

Assim, verifica-se que, utilizando-se o modelo proposto, tanto o ponto de máximo carregamento, quanto a impedância equivalente neste ponto, variam quando o tap é alterado, independente do sentido do fluxo de potência, conforme intuição, comprovada através de ensaios em laboratório (Ferreira e Prada, 2009), apresentados no Apêndice A.

\section{EFEITOS DE AÇÕES DE CONTROLE DE TENSÃO: COMPARAÇÃO ENTRE OS MODELOS USUAL E PROPOSTO}

Analisando-se ainda o sistema de 2 barras, considera-se agora, uma troca de tap, de $\mathrm{a}_{1}$ para $\mathrm{a}_{2}$, com o objetivo de aumentar a tensão no lado $k$, onde está localizada a carga

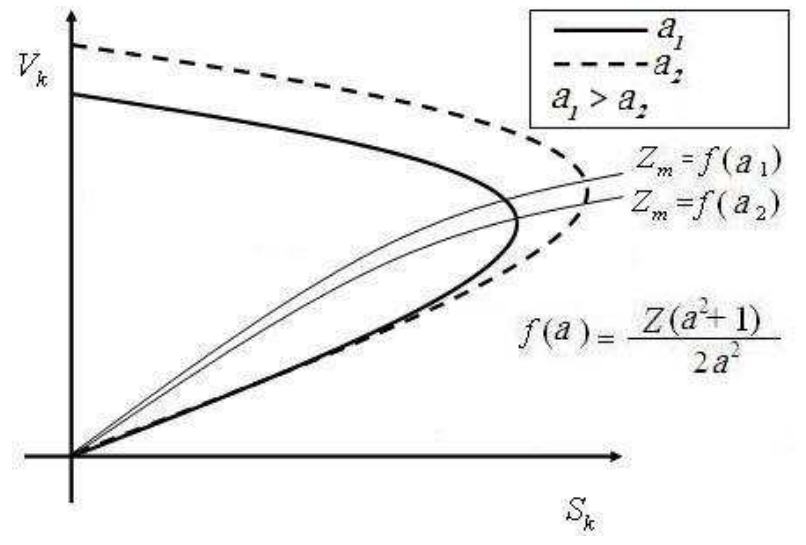

Figura 17: Curvas $\varphi$ e Z Constantes para Dois Valores de tap com o Modelo Proposto e com Fluxo da barra $m$ para a barra $k$

(fluxo de potência da barra $m$ para a barra $k$ ). Essa troca corresponde, então, a um decremento no valor do tap, que está do lado $m$. Considera-se também que o ponto de operação analisado está na região anormal, como apresentado na Figura 18. Utilizando-se o modelo usual, verifica-se que a ação de controle de tensão (redução do tap) faz com que o valor da tensão aumente, se o modelo da carga é potência constante. Por outro lado, se o modelo da carga é impedância constante, a tensão diminui, conforme (Baracho e Café, 1991; El-Sadek et alii, 1999; IEEE/PES, 2002).

Resultados opostos são obtidos utilizando-se o modelo proposto. Ou seja, ao reduzir o tap, o valor da tensão diminui, caso o modelo da carga seja potência constante e, se o modelo da carga é impedância constante, a tensão aumenta. Destaca-se, ainda, que os resultados obtidos com o modelo proposto, no que tange ao controle de tensão, também são independentes do sentido do fluxo de potência e estão coerentes com o que foi apresentado na Seção 2.1.2, ao contrário do modelo usual.

\section{RESULTADOS}

Nas simulações apresentadas a seguir, foi efetuado carregamento adicional em todas as barras com carga dos sistemas, mantendo-se constante o fator de potência. O aumento da carga é suprido apenas pelo gerador da barra de referência de ângulo de tensão.

Inicialmente, será apresentado resultado de simulação na qual os taps dos transformadores são mantidos com valores diferentes do valor nominal, não sendo efetuado controle de tensão. Posteriormente, os transformadores controlarão tensões de barras, através da variação do tap. As simulações fo- 


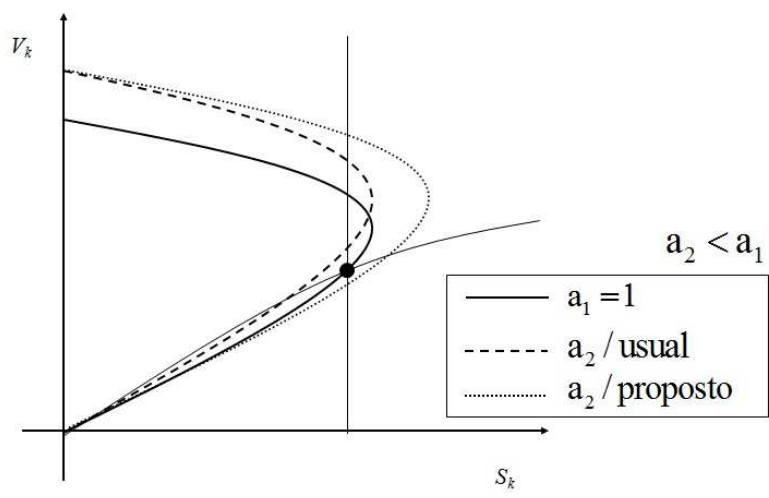

Figura 18: Comparação entre os Modelos Usual e Proposto para Fluxo da barra $m$ para barra $k$

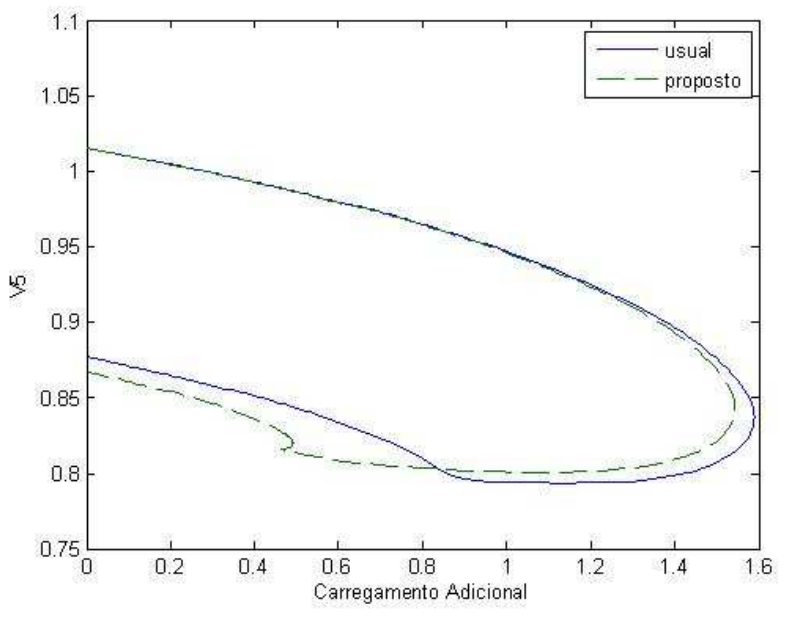

Figura 19: Comparação entre os Modelos Usual e Proposto / Barra 5 / IEEE14 Barras

\subsection{Sistema IEEE-57 Barras}

Neste sistema-teste, o transformador situado entre as barras 32 e 34 controla a tensão na barra 32, enquanto o transformador situado entre as barras 10 e 51 controla remotamente a tensão da barra 50 .

As curvas $\phi$ constante apresentadas nas Figuras 21 e 22 foram obtidas através de um algoritmo de Fluxo de Potência Continuado com possibilidade de se controlar tensões de barras terminais ou remotas através da variação de tap de transformador.

Observa-se que a tensão da barra 32 é controlada até o tap do transformador atingir seu limite máximo de 1,15 p.u. A partir do ponto em que o limite máximo é atingido, a tensão dessa barra não volta mais a ser controlada. Diferenças entre os dois modelos podem ser observadas: i) no carregamento no qual o tap máximo é atingido, de forma que, para carregamento adicional em torno de 0,08 p.u., a tensão é controlada ou não dependendo do modelo; ii) nos valores diferentes das tensões para cada carregamento adicional e; iii) no ponto de máximo carregamento, que é inferior com o modelo proposto.

\subsection{Sistema Teste Brasileiro de 33 Barras}

Diferenças entre os dois modelos também foram notadas nos valores dos fluxos de potência, sendo bastante significativas em algumas simulações. Comparando-se os valores dos fluxos de potência do caso-base do sistema-teste brasileiro de 33 barras, apresentado em (STB, 2011), além de grande diferença quantitativa referente ao valor do fluxo de potência ativa entre as barras 896 e 897 (-279,6 MW pelo 


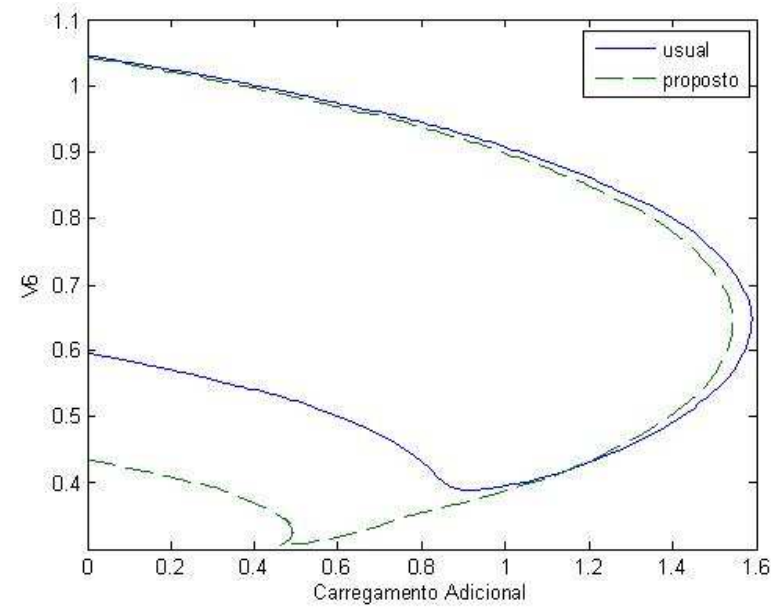

Figura 20: Comparação entre os Modelos Usual e Proposto / Barra 6 / IEEE14 Barras

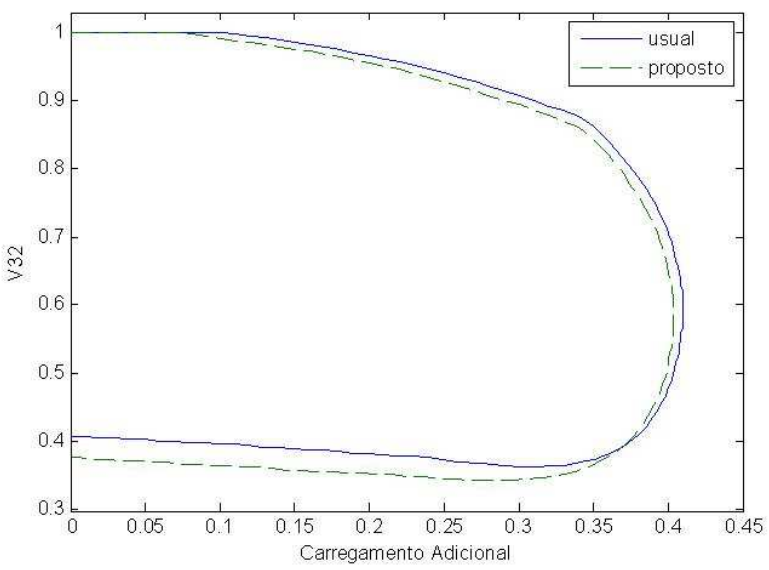

Figura 21: Comparação Entre os Modelos Usual e Proposto / Barra 32 / Controle de Tensão / IEEE 57 Barras

modelo usual e 23,9 MW pelo modelo proposto), observase, também, diferença qualitativa, uma vez que os sentidos dos fluxos informados pelos dois modelos são opostos. Com relação aos valores de tensão no caso-base, diferenças também podem ser notadas. Como exemplo, a tensão da barra 896 (terminal de transformador com tap variável) vale $0,982 \mid 4,9^{\circ}$ p.u. pelo modelo usual e

$1,0 0 1 \longdiv { 1 2 , 4 ^ { \circ } }$ p.u. pelo modelo proposto.

\section{CONCLUSÕES}

Foi proposto um novo modelo de transformador com tap variável, mais próximo da realidade física do equipamento, e adequado para estudos de estabilidade de tensão.

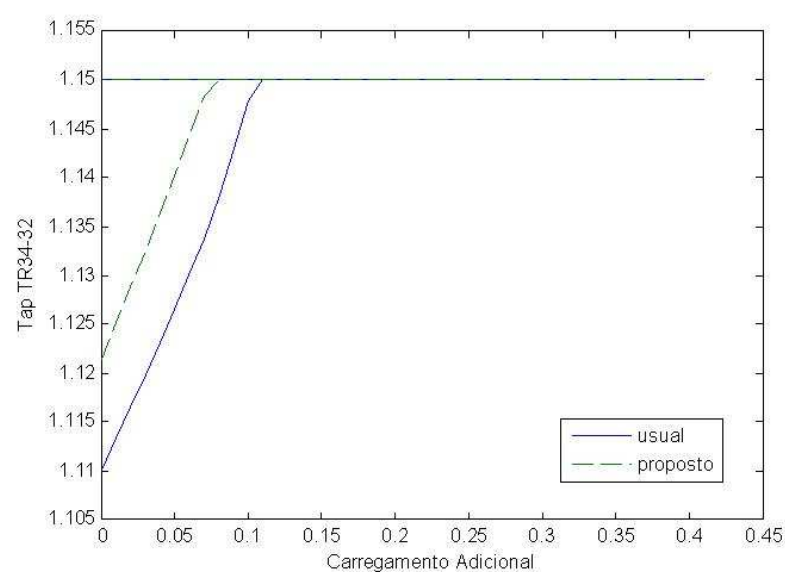

Figura 22: Comparação entre os Modelos Usual e Proposto / Transformador entre as Barras 32 e 34 / Controle de Tensão / IEEE 57 Barras

Ao contrário do modelo usual, a impedância referente a determinado lado é variável com o tap. Assim, pode-se avaliar corretamente o efeito da troca de tap sobre a tensão a ser controlada e sobre a margem de potência até o máximo carregamento.

Quando se analisa a necessidade de bloquear a ação de transformadores com taps variáveis para evitar a possibilidade de efeito do controle de tensão oposto ao esperado, como apresentado em (Zambroni de Souza e De Lima Lopes, 2003), é necessário verificar a influência dessa atitude na margem de potência. Assim, é fundamental o uso do modelo proposto, uma vez que margens diferentes são obtidas com os dois modelos.

O modelo proposto se aplica a qualquer estudo de regime permanente com transformador de tap variável. Através de diversas simulações computacionais, diferenças quantitativas e principalmente qualitativas foram obtidas comparando-se os resultados dos dois modelos.

\section{REFERÊNCIAS BIBLIOGRÁFICAS}

Ajjarapu, V e Christy, C. (1992). The Continuation Power Flow: a Tool for Steady State Voltage Stability Analysis. IEEE Transactions on Power Systems (February), Vol. 7, No. 1, pp. 416:423.

Baracho, F.R.A. e Café, F.F. (1991). Análise de Instabilidade de Tensão em Sistema Elétricos. XI SNPTEE, Rio de Janeiro-RJ.

Boyajian, A. (1929). New Theory of Transformer and Autotransformer Circuits. General Electric Review (February), Vol 32, p. 110-119. 
Boyajian, A. (1930). Inversion Currents and Voltages in Auto-Transformers. AIEE Transactions (April), Vol 49, p. $810-818$.

Boyajian, A. (1930). Inversion Currents and Voltages in Auto-Transformers, Discussion by I.H. Summers. AIEE Transactions (April), Vol 49, p. 819.

Clarke, E. (1950). Circuit Analysis of A-C Power Systems, Ed. John Wiley \& Sons, Vol 2.

Close, C.M. (1975). Circuitos Lineares, Ed. 2 LTC S.A..

Del Toro, V. (1990). Basic Electric Machines, Ed. Prentice Hall.

El-Sadek, M.Z et alii (1999). Tap Changing Transformer Role in Voltage Stability Enhancement. Electric Power System Research 50, p. 115-118.

Ferreira, C.A. e Prada, R.B. (2009). Avaliação do Modelo Atual de Transformador com Tap Variável. VIII CLAGTEE, Ubatuba-SP.

Grainger, J.J. and Stevenson Jr, W.D. (1994). Power System Analysis, New York, McGraw-Hill.

IEEE/PES 2002. Voltage Stability Assessment: Concepts, Practices and Tools, Cap 2: Basic Theoretical Concepts (Final Document).

MacLeod, D.R. (1929). New Equivalent Circuits for Autotransformer and Transformer Circuits. General Electric Review (February), Vol 32, p. 120-126.

Monticelli, A.J. e Garcia A. (2003). Introdução a Sistemas de Energia Elétrica, Editora da Unicamp.

PSTCA (2011) - Power System Test Case Archive, Disponível no sítio: http://www.ee.washington.edu/research/pstca Acessado no dia 08/01/2011.

Staff of MIT - Members of the Staff of the Department of Electrical Engineering Massachussets Institute of Technology. (1965). Magnetic Circuits and Transformers, Ed. 15, Cambridge: MIT Press.

STB (2011) - Sistemas-Teste Brasileiros para Uso Didático e Análise Computacional de Sistemas Elétricos de Potência, Disponível no sítio: http://www.sistemasteste.com.br/. Acessado no dia 08/01/2011.

Westinghouse (1964) - Central Station Engineers of the Westinghouse Electric Corporation. Electrical Transmission and Distribution Reference Book, Ed. 4, East Pittsburg.
Zambroni de Souza, A.C. e de Lima Lopes, B.I. (2003). On Multiple Tap Blocking to Avoid Voltage Collapse. Electric Power Systems Research, Vol 67, n. 3, p. 225231.

\section{APÊNDICE A - ENSAIOS EM LABORATÓ- RIO}

Como o objetivo de se verificar os efeitos reais da variação do tap de um transformador, para cada sentido do fluxo de potência através do mesmo, foram realizados ensaios em laboratório com um transformador de 1kVA, 220:220V (corrente nominal de 4,5A), com taps em 190 e $110 \mathrm{~V}$ em ambos os lados.

Os ensaios consistiram na alimentação de um dos lados do transformador e inserção gradativa de resistores no outro lado. Variação do tap e do sentido do fluxo de potência foram efetuadas, de forma a confirmar a inconsistência do modelo usual e a validade do modelo proposto de transformador com tap variável.

\section{A.1 Fluxo de Potência do Lado de Baixa para o Lado de Alta Tensão}

Nessa situação, o lado de baixa tensão foi alimentado com tensão nominal $(110 \mathrm{~V})$, sendo inserida carga no lado de alta tensão gradativamente. Para cada inserção de carga foram anotados os valores da potência e da tensão na carga. Esse procedimento foi realizado para os dois taps do transformador, ou seja, para as situações em que as relações de transformação são 110:190V e 110:220V. A Figura A.1 apresenta as duas curvas $\varphi$ constante no plano PV, uma para cada valor de tap.

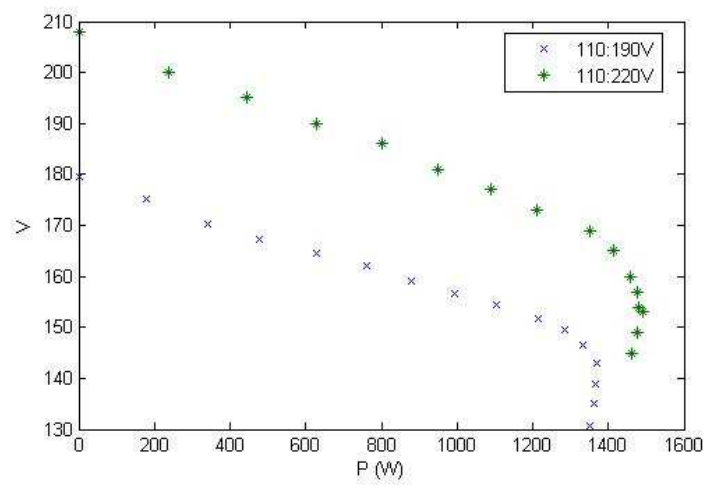

Figura A.1: Curvas $\varphi$ Constante para Dois Valores de Tap Obtidas em Laboratório e com Fluxo de Potência do Lado de Baixa para o Lado de Alta Tensão 
De acordo com a Figura A.1, verifica-se que, aumentandose o tap do transformador, o ponto de máximo carregamento varia de $1370 \mathrm{~W}$ para $1490 \mathrm{~W}$. A partir de medições de correntes realizadas nos pontos de máximo carregamento, foi possível, também, obter a impedância equivalente da carga nesses pontos, tendo sido verificado que a mesma aumentou de $17,4 \Omega$ para $18,4 \Omega$. É importante mencionar que, após a obtenção do ponto de máximo carregamento, foi interrompido o teste, devido à crescente dissipação de potência no transformador desde vazio até curto-circuito.

\section{A.2 Fluxo de Potência do Lado de Alta para o Lado de Baixa Tensão}

Nesse caso, o lado de alta tensão foi alimentado com 220 $\mathrm{V}$, sendo inserida carga no lado de baixa tensão gradativamente. Novamente, para cada inserção de carga foram anotados os valores da potência e da tensão na carga. Esse procedimento foi realizado para os dois taps do transformador, ou seja, para as situações em que as relações de transformação são 190:110V e 220:110V. Na Figura A.2 apresenta-se as 2 curvas $\varphi$ constante no plano PV, uma para cada valor de tap.

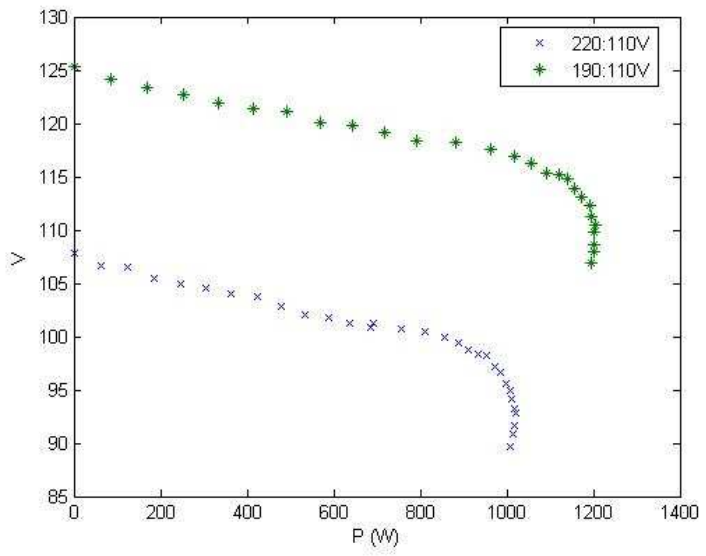

Figura A.2: Curvas $\varphi$ Constante para Dois Valores de Tap Obtidas em Laboratório e com Fluxo de Potência do Lado de Alta para o Lado de Baixa Tensão

De acordo com a Figura A.2, verifica-se que, diminuindose o tap do transformador, o ponto de máximo carregamento varia de $1022 \mathrm{~W}$ para $1205 \mathrm{~W}$. A partir de medições de correntes realizadas nos pontos de máximo carregamento, foi possível, também, obter a impedância equivalente da carga nesses pontos, tendo sido verificado que a mesma aumentou de $10,4 \Omega$ para $12,3 \Omega$.

Dos ensaios realizados confirmou-se que, tanto a impedância equivalente da carga no ponto de máximo carregamento, quanto a margem de estabilidade de tensão, variam quando o tap é alterado, independente do sentido do fluxo de potência, conforme o modelo proposto de transformador com tap variável. 